\title{
A general stability result for second order stochastic quasilinear evolution equations with memory
}

\author{
Hui Yang ${ }^{1,2}$, Shiyue Fang ${ }^{3}$, Fei Liang ${ }^{2 *}$ and Min Li
}

\author{
"Correspondence: \\ fliangmath@126.com; \\ liangfei@xust.edu.cn \\ 2Department of Mathematics, Xi An \\ University of Science and \\ Technology, Xi An, China \\ Full list of author information is \\ available at the end of the article
}

\begin{abstract}
The goal of this paper is to discuss an initial boundary value problem for the stochastic quasilinear viscoelastic evolution equation with memory driven by additive noise. We prove the existence of global solution and asymptotic stability of the solution using some properties of the convex functions. Moreover, our result is established without imposing restrictive assumptions on the behavior of the relaxation function at infinity.
\end{abstract}

MSC: $60 \mathrm{H} 15 ; 35 \mathrm{~L} 05 ; 35 \mathrm{~L} 70$

Keywords: Quasilinear stochastic viscoelastic wave equations; Explosive solutions; Energy inequality

\section{Introduction}

The quasilinear viscoelastic wave equation of the following form:

$$
\left\{\begin{array}{l}
\left|u_{t}\right|^{\rho} u_{t t}-\Delta u+\int_{0}^{t} g(t-s) \Delta u(s) d s+h\left(u_{t}\right)=f(u), \quad(x, t) \in D \times(0, T) \\
u(x, t)=0, \quad(x, t) \in \partial D \times(0, T), \\
u(x, 0)=u_{0}(x), \quad u_{t}(x, 0)=u_{1}(x), \quad x \in D
\end{array}\right.
$$

describes a viscoelastic material, with $u(x, t)$ giving the position of material particle $x$ at time $t$, where $D$ is a bounded domain in $\mathbb{R}^{d}$ with a smooth boundary $\partial D, \rho>0, g$ is the relaxation function, $f$ denotes the body force, and $h$ is the damping term. The properties of the solution to (1.1) have been studied by many authors (see [1-7]). For instance, in [1], Cavalcanti et al. considered (1.1) for $h\left(u_{t}\right)=-\gamma \Delta u_{t}$ and $f(u)=0$, where $0<\rho \leq 2 /(d-$ $2)$ if $d \geq 3$ or $\rho>0$ if $d=1,2$. They proved a global existence result when the constant $\gamma \geq 0$ and an exponential decay result for the case $\gamma>0$. Messaoudi et al. [4] studied (1.1) for $h\left(u_{t}\right)=-\Delta u_{t t}$ and $f(u)=0$, they proved an explicit and general decay rate result with some properties of the convex functions. Liu [5] considered (1.1) for $h\left(u_{t}\right)=0$ and $f(u)=b|u|^{p-2} u$, where $b>0, p>2$. The author proved that, for a certain class of relaxation functions and certain initial data in the stable set, the decay rate of the solution energy is similar to that of the relaxation function. Conversely, he also obtained for certain initial

(c) The Author(s) 2020. This article is licensed under a Creative Commons Attribution 4.0 International License, which permits use, sharing, adaptation, distribution and reproduction in any medium or format, as long as you give appropriate credit to the original author(s) and the source, provide a link to the Creative Commons licence, and indicate if changes were made. The images or other third party material in this article are included in the article's Creative Commons licence, unless indicated otherwise in a credit line to the material. If material is not included in the article's Creative Commons licence and your intended use is not permitted by statutory regulation or exceeds the permitted use, you will need to obtain permission directly from the copyright holder. To view a copy of this licence, visit http://creativecommons.org/licenses/by/4.0/. 
data in the unstable set that there are solutions that blow up in finite time. In [6], Song investigated (1.1) for $h\left(u_{t}\right)=\left|u_{t}\right|^{q-2} u_{t}$ and $f(u)=|u|^{p-2} u$, where $q>2$, and $\rho, p$ satisfy

$$
\begin{cases}2<p<\infty, \quad 2<\rho<\infty, & \text { if } d=1,2, \\ 2<p<2(d-1) /(d-2), & 2<\rho \leq d /(d-2), \quad \text { if } d \geq 3 .\end{cases}
$$

He proved the global nonexistence of the positive initial energy solutions of the quasilinear viscoelastic wave equation. Cavalcanti et al. [7] also studied (1.1) with $h\left(u_{t}\right)=a(x) u_{t}$ and $f(u)=b|u|^{p-2} u$, where $a(x)$ can be null on a part of the boundary, they obtained an exponential rate of decay of solutions.

In fact, the driving force may be affected by the random environment. In view of this, we consider the following stochastic quasilinear viscoelastic wave equations:

$$
\left\{\begin{array}{l}
\left|u_{t}\right|^{\rho} u_{t t}-\Delta u-\Delta u_{t t}+\int_{0}^{t} g(t-s) \Delta u d s=\sigma(x, t) \partial_{t} W(t, x), \quad \text { in } D \times(0, \infty) \\
u(x, t)=0, \quad \text { on } \Gamma \times(0, \infty), \\
u(x, 0)=u_{0}(x), \quad u_{t}(x, 0)=u_{1}(x), \quad \text { in } D
\end{array}\right.
$$

where $g$ is a positive function satisfying some conditions to be specified later, $\sigma$ is local Lipschitz continuous, $W(t, x)$ is an infinite dimensional Wiener random field, and the initial data $u_{0}(x)$ and $u_{1}(x)$ are $\mathcal{F}_{0}$-measurable given functions.

To motivate our work, let us firstly recall some results regarding $\rho=0$ and $g \equiv 0$, then (1.2) can be rewritten as the following stochastic wave equation:

$$
u_{t t}-\Delta u+h\left(u_{t}\right)=f(u)+\sigma(x, t) \partial_{t} W(t, x), \quad x \in D, t \in(0, T) .
$$

In $[8,9]$, Chow considered the large-time asymptotic properties of solutions to a class of semi-linear stochastic wave equations with linear damping in a bounded domain. Under appropriate conditions, the author obtained the exponential stability of an equilibrium solution in mean-square and the almost sure sense by energy inequality. Using Lyapunov function techniques, Brzeźniak et al. [10] proved global existence and stability of solutions for the stochastic nonlinear beam equations. In [11], Brzeźniak and Zhu studied a type of stochastic nonlinear beam equation with locally Lipschitz coefficients. Using a suitable Lyapunov function and applying the Khasminskii test they showed the nonexplosion of the mild solutions. In addition, under some additional assumptions they proved the exponential stability of the solution. Kim [12] and Barbu et al. [13] investigated initial boundary value stochastic wave equations with nonlinear damping and dissipative damping, respectively. There are also many results on the stochastic wave equations, see the references in $[10,14-20]$.

When $\rho=0$ and $g \neq 0,(1.2)$ can be rewritten as the following stochastic viscoelastic wave equation:

$$
u_{t t}-\Delta u+\int_{0}^{t} g(t-s) \Delta u d s+h\left(u_{t}\right)=f(u)+\varepsilon \sigma(x, t) \partial_{t} W(t, x), \quad x \in D, t \in(0, T) .
$$

For the current equation (1.4), the memory part makes it difficult to estimate the energy by using these methods which are used in stochastic wave equation. Hence, Wei and Jiang 
[21] studied (1.4) with $\sigma \equiv 1$ and $q=2$ in another way. They showed the existence and uniqueness of solution for (1.4) and obtained the decay estimate of the energy function of the solution under some appropriate assumption on $g$. In [22], Liang and Gao extended the existence and uniqueness results of [21] with $\sigma=\sigma(u, \nabla u, x, t)$. In the case of $\sigma=\sigma(x, t)$, they proved that the solution either blows up in finite time with positive probability or is explosive in $L^{2}$ using the energy inequality. Furthermore, Liang and Gao [23] considered (1.4) driven by Lévy noise, they proved the global existence and uniqueness of the mild solution with the appropriate energy function and obtained the exponential stability of the solutions. Liang and Guo [24] studied (1.4) driven by multiplicative noise, the authors proved the global existence and asymptotic stability of the mild solution by the Lyapunov function.

Furthermore, Kim et al. [25] considered (1.2) with $\rho \neq 0$ and $g \neq 0$ driven by an additive noise, i.e., $\sigma=\sigma(x, t)$. By an appropriate energy inequality, they proved that finite time blow-up is possible for equation (1.2) if $p>\{q, \rho+2\}$ and the initial energy is sufficiently negative.

We note that in the above literature, Messaoudi et al. [4], Liang and Gao [23], Chen et al. [24], and Kim et al. [25] did not discuss the optimality of the decay rate of (1.2) under the influence of random environment. We prove the stability of solutions to (1.2) by modifying the convex functions. The result of this paper provides an explicit energy decay formula that allows a larger class of functions $g$ from which the energy decay rates are not necessarily of exponential or polynomial types.

This paper is organized as follows. In Sect. 2, we present some assumptions and definitions needed for our work. Section 3 shows the statement and proof of our main result.

\section{Preliminaries}

Firstly, let us introduce some notations used throughout this paper. We set $H=L^{2}(D)$ with the inner product and norm denoted by $(\cdot, \cdot)$ and $\|\cdot\|_{2}$, respectively. Denote by $\|\nabla \cdot\|_{2}$ the Dirichlet norm in $V=H_{0}^{1}(D)$. We consider the following hypotheses.

(A1) $\rho, p, q$ satisfy

$$
\left\{\begin{array}{l}
0<\rho \leq \frac{2}{d-2}, \quad \text { if } d \geq 3, \\
\rho>0, \quad \text { if } d=1,2
\end{array}\right.
$$

(A2) $g \in C^{1}[0, \infty)$ is a nonnegative and nonincreasing function satisfying

$$
1-\int_{0}^{\infty} g(s) d s=l>0
$$

(A3) There exists a positive function $H \in C^{1}(\mathbb{R}+)$, with $H(0)=0$, and $H$ is a linear or strictly increasing and strictly convex $C^{2}$ function on $(0, r]$ for some $r<1$ such that

$$
g^{\prime}(t) \leq-H(g(t)), \quad \forall t \geq 0 .
$$

Definition 2.1 Assume that $\left(u_{0}, u_{1}\right) \in H_{0}^{1}(D) \times L^{2}(D)$ and $\mathbf{E} \int_{0}^{T}\|\sigma(t)\|^{2} d t<\infty, u$ is said to be the solution of (1.2) on the interval [0,T], if $\left(u, u_{t}\right)$ is $H_{0}^{1}(D) \times L^{2}(D)$-valued progressively measurable, $\left(u, u_{t}\right) \in L^{2}\left(\Omega ; C\left([0, T] ; H_{0}^{1}(D) \times L^{2}(D)\right)\right), u_{t} \in L^{q}((0, T) \times D)$, and such that (1.2) holds in the sense of distributions over $(0, T) \times D$ for almost all $\omega$. 
Theorem 2.1 Assume that $\left(u_{0}, u_{1}\right) \in H_{0}^{1}(D) \times L^{2}(D), \mathbf{E} \int_{0}^{T}\|\sigma(t)\|^{2} d t<\infty$, and condition (2.1) holds. $u_{t}$ is a solution of (1.2) with initial data $\left(u_{0}, u_{1}\right) \in H_{0}^{1}(D) \times L^{2}(D)$, according to Definition 2.1 on the interval $[0, T]$, for any $T>0$, we have

$$
\mathbf{E} \sup _{0 \leq t \leq T} e(u(t))<\infty
$$

Similar to Theorem 4.1 of [25], we can explicitly drive the proof of the above theorem. Now, we introduce the "modified" energy associated with problem (1.2):

$$
\mathcal{E}(t)=\frac{1}{\rho+2}\left\|u_{t}(t)\right\|_{\rho+2}^{\rho+2}+\frac{1}{2} l\|\nabla u(t)\|_{2}^{2}+\frac{1}{2}\left\|\nabla u_{t}(t)\right\|_{2}^{2}+\frac{1}{2}(g \circ \nabla u)(t),
$$

where, for any $w \in L^{2}(D)$,

$$
(g \circ w)(t)=\int_{0}^{t} g(t-s)\|w(t)-w(s)\|_{2}^{2} d s
$$

Let $(\Omega, P, \mathcal{F})$ be a complete probability space for which $\left\{\mathcal{F}_{t}, t \geq 0\right\}$ of sub- $\sigma$-fields of $\mathcal{F}$ is given. A point of $D$ will be denoted by $D$ and $\mathbf{E}(\cdot)$ stands for expectation with respect to probability measure $P$. When $\mathcal{O}$ is a topological space, $\mathcal{B}$ denotes the Borel $\sigma$-algebra over $\mathcal{O}$. Suppose that $\{W(t, x): t \geq 0\}$ is a $H$-value $Q$-Wiener process on the probability space with the variance operator $Q$ satisfying $\operatorname{Tr} Q<\infty$. Moreover, we can assume that $Q$ has the following form: $Q e_{i}=\lambda_{i} e_{i}, i=1,2, \ldots$, where $\lambda_{i}$ are eigenvalues of $Q$ satisfying $\sum_{i=1}^{\infty} \lambda_{i}<\infty$ and $\left\{e_{i}\right\}$ are the corresponding eigenfunctions with $c_{0}:=\sup _{i \geq 1}\left\|e_{i}\right\|_{\infty}<\infty\left(\|\cdot\|_{\infty}\right.$ denotes the super-norm). To simplify the computations, we assume that the covariance operator $Q$ and $-\Delta$ with homogeneous Dirichlet boundary condition have a common set of eigenfunctions, i.e., $\left\{e_{i}\right\}_{i=1}^{\infty}$ satisfy

$$
\left\{\begin{array}{l}
-\Delta e_{i}=\mu_{i} e_{i}, \quad x \in D \\
e_{i}=0, \quad x \in \partial D
\end{array}\right.
$$

and form an orthonormal base of $H$. In this case, $W(t, x)=\sum_{i=1}^{\infty} \sqrt{\lambda_{i}} B_{i}(t) e_{i}$, where $\left\{B_{i}(t)\right\}$ is a sequence of independent copies of standard Brownian motions in one dimension. In addition, $\{W(t, x): t \geq 0\}$ is an $H$-valued $Q$-Wiener process. For more details about the infinite-dimensional Wiener process and the stochastic integral, see in [26, 27].

\section{Stability properties of solutions}

In this section, we state and prove our main stability result. Throughout this section, we suppose that $\sigma(x, t, w)=\sigma(x, t)$ such that

$$
\int_{0}^{\infty} \int_{D} \sigma^{2}(x, t) d x d t<\infty
$$

As is well known, equation (1.2) is equivalent to the following Itô system:

$$
\left\{\begin{array}{l}
d u=v d t, \\
d\left(\frac{1}{\rho+1}|v|^{\rho} v\right)=\left(\Delta u+\Delta u_{t t}-\int_{0}^{t} g(t-s) \Delta u(s) d s\right) d t+\sigma(x, t) d W(t, x) .
\end{array}\right.
$$


In order to prove our stability result, we need the following lemmas.

Lemma 3.1 Let $u_{0}(x)$ and $u_{1}(x)$ be $\mathcal{F}_{0}$-measurable with $u_{0}(x) \in H_{0}^{1}(D)$ and $u_{1}(x) \in L^{2}(D)$. Assume (2.1) holds. Let $(u, v)$ be a solution of system (3.2). Then we have

$$
\begin{aligned}
\frac{d}{d t} \mathbf{E} \mathcal{E}(t) & =\frac{1}{2} \sum_{i=1}^{\infty} \mathbf{E} \int_{D} \lambda_{i} e_{i}^{2}(x) \sigma^{2}(x, t) d x-\frac{1}{2} \mathbf{E}\left(-g^{\prime} \circ \nabla u\right)(t)-\frac{1}{2} g(t) \mathbf{E}\|\nabla u(t)\|_{2}^{2} \\
& \leq \frac{1}{2} \sum_{i=1}^{\infty} \mathbf{E} \int_{D} \lambda_{i} e_{i}^{2}(x) \sigma^{2}(x, t) d x+\frac{1}{2} \mathbf{E}\left(g^{\prime} \circ \nabla u\right)(t) .
\end{aligned}
$$

Proof Applying Itô's formula to $\frac{2}{\rho+2}\|v\|_{\rho+2}^{\rho+2}$, we get

$$
\begin{aligned}
\frac{2}{\rho+2}\|v\|_{\rho+2}^{\rho+2}= & \frac{2}{\rho+2}\left\|v_{0}\right\|_{\rho+2}^{\rho+2}-2 \int_{0}^{t}(\nabla u, \nabla v) d s+2 \int_{D}\left(\int_{0}^{t} g(t-s) \nabla u(s) d s, \nabla v(t)\right) d x \\
& -2 \int_{0}^{t}\left(\nabla u_{t t}, \nabla v\right) d s+2 \int_{0}^{t}\left(v, \sigma(x, s) d W_{s}\right)+\int_{0}^{t}\|\sigma(x, s)\|_{L_{2}^{0}}^{2} d s \\
= & 2 \mathbf{E} \mathcal{E}(0)-\|\nabla u(t)\|_{2}^{2}-\left\|\nabla u_{t}(t)\right\|_{2}^{2}+2 \int_{D}\left(\int_{0}^{t} g(t-s) \nabla u(s) d s, \nabla v(t)\right) d x \\
& +2 \int_{0}^{t}\left(v, \sigma(x, s) d W_{s}\right)+\int_{0}^{t}\|\sigma(x, s)\|_{L_{2}^{0}}^{2} d s .
\end{aligned}
$$

For the third term on the right-hand side of (3.4), we obtain

$$
\begin{aligned}
& 2\left(\int_{0}^{t} g(t-s) \nabla u(s) d s, v(t)\right) \\
& \quad=2 \int_{0}^{t} g(t-s) \int_{D} \nabla v(t)(\nabla u(s)-\nabla u(t)) d x d s+2 \int_{0}^{t} g(t-s) \int_{D} \nabla v(t) \nabla u(t) d x d s \\
& \quad=-\int_{0}^{t} g(t-s) \frac{d}{d t} \int_{D}|\nabla u(s)-\nabla u(t)|^{2} d x d s+\int_{0}^{t} g(s) \frac{d}{d t} \int_{D}|\nabla u(t)|^{2} d x d s \\
& \quad=\frac{d}{d t}\left(\int_{0}^{t} g(s) d s\|\nabla u(t)\|_{2}^{2}-(g \circ \nabla u)(t)\right)+\left(g^{\prime} \circ \nabla u\right)(t)-g(t)\|\nabla u(t)\|_{2}^{2} .
\end{aligned}
$$

Inserting (3.5) into (3.4) and taking the expectation for (3.4), we get (3.3).

Let

$$
S(t)=\frac{1}{2} \sum_{i=1}^{\infty} \mathbf{E} \int_{0}^{t} \int_{D} \lambda_{i} e_{i}^{2}(x) \sigma^{2}(x, s) d x d s .
$$

From (3.1), we have

$$
\begin{aligned}
S(\infty) & =\frac{1}{2} \sum_{i=1}^{\infty} \mathbf{E} \int_{0}^{\infty} \int_{D} \lambda_{i} e_{i}^{2}(x) \sigma^{2}(x, s) d x d s \\
& \leq \frac{1}{2} c_{0}^{2} \operatorname{Tr} R \mathbf{E} \int_{0}^{\infty} \int_{D} \sigma^{2}(x, s) d x d s \\
& =E_{1}<\infty .
\end{aligned}
$$


Then we integrate (3.3) on $(0, T)$, which yields

$$
\mathbf{E} \mathcal{E}(t) \leq \mathbf{E} \mathcal{E}(0)+E_{1}
$$

Lemma 3.2 Let $u$ be a solution of (1.2). The functional

$$
\Psi(t):=\frac{1}{\rho+1} \int_{D}\left|u_{t}\right|^{\rho} u_{t} u d x+\int_{D} \nabla u \cdot \nabla u_{t} d x
$$

satisfies, along the solution of (1.2), the estimate

$$
\begin{aligned}
\mathbf{E} \Psi^{\prime}(t) \leq & -\frac{l}{2} \mathbf{E} \int_{D}|\nabla u|^{2} d x+\mathbf{E} \int_{D}\left|\nabla u_{t}\right|^{2} d x+\frac{1}{\rho+1} \mathbf{E} \int_{D}\left|u_{t}\right|^{\rho+2} d x \\
& +\frac{1-l}{2 l} \mathbf{E}(g \circ \nabla u)(t) .
\end{aligned}
$$

Proof Direct differentiation of $\Psi$, using (1.2), yields

$$
\begin{aligned}
\Psi^{\prime}(t)= & -\int_{D}|\nabla u|^{2} d x+\int_{D} \nabla u(t) \cdot \int_{0}^{t} g(t-s) \nabla u(s) d s d x+\int_{D}\left|\nabla u_{t}\right|^{2} d x \\
& +\frac{1}{\rho+1} \int_{D}\left|u_{t}\right|^{\rho+2} d x+\int_{D}\left(\sigma(x, s) d W_{s}, u\right) d x .
\end{aligned}
$$

We take the expectation of the above formula to get the following result:

$$
\begin{aligned}
\mathbf{E} \Psi^{\prime}(t)= & -\mathbf{E} \int_{D}|\nabla u|^{2} d x+\mathbf{E} \int_{D} \nabla u(t) \cdot \int_{0}^{t} g(t-s) \nabla u(s) d s d x \\
& +\mathbf{E} \int_{D}\left|\nabla u_{t}\right|^{2} d x+\frac{1}{\rho+1} \mathbf{E} \int_{D}\left|u_{t}\right|^{\rho+2} d x
\end{aligned}
$$

for any general solution. With simple density parameters, this estimate is still applicable for weak solutions. Then we estimate that the second item on the right-hand side of (3.9) is as follows:

$$
\begin{aligned}
& \int_{D} \nabla u(t) \cdot \int_{0}^{t} g(t-s) \nabla u(s) d s d x \\
& \quad \leq \frac{1}{2} \int_{D}|\nabla u|^{2} d x+\frac{1}{2} \int_{D}\left(\int_{0}^{t} g(t-s)(|\nabla u(t)-\nabla u(s)|+|\nabla u(t)|) d s\right)^{2} d x .
\end{aligned}
$$

By using

$$
\int_{0}^{t} g(s) d s<\int_{0}^{\infty} g(s) d s=1-l
$$

and

$$
(a+b)^{2} \leq(1+\eta) a^{2}+\left(1+\frac{1}{\eta}\right) b^{2}, \quad \forall \eta>0,
$$

we arrive at

$$
\int_{D} \nabla u(t) \cdot \int_{0}^{t} g(t-s) \nabla u(s) d s d x
$$




$$
\begin{aligned}
\leq & \frac{1}{2} \int_{D}|\nabla u|^{2} d x+\frac{1}{2}(1+\eta)(1-l)^{2} \int_{D}|\nabla u|^{2} d x \\
& +\frac{1}{2}\left(1+\frac{1}{\eta}\right) \int_{D}\left(\int_{0}^{t} g(t-s)|\nabla u(t)-\nabla u(s)| d s\right)^{2} d x \\
\leq & \frac{1}{2}\left[1+(1+\eta)(1-l)^{2}\right] \int_{D}|\nabla u|^{2} d x+\frac{1}{2}\left(1+\frac{1}{\eta}\right)(1-l)(g \circ \nabla u)(t) .
\end{aligned}
$$

By taking $\eta=\frac{l}{1-l}$, we obtain

$$
\int_{D} \nabla u(t) \cdot \int_{0}^{t} g(t-s) \nabla u(s) d s d x \leq \frac{2-l}{2} \int_{D}|\nabla u|^{2} d x+\frac{1-l}{2 l}(g \circ \nabla u)(t) .
$$

Inserting (3.11) in (3.9), we get (3.8).

Lemma 3.3 Let $u$ be a solution of (1.2). The functional

$$
\chi(t):=\int_{D}\left(\Delta u_{t}-\frac{\left|u_{t}\right|^{\rho} u_{t}}{\rho+1}\right) \int_{0}^{t} g(t-s)(u(t)-u(s)) d s d x
$$

satisfies the solution of equation (1.2) and, for any $\delta_{1}, \delta_{2}>0$, the estimate

$$
\begin{aligned}
\mathbf{E} \chi^{\prime}(t) \leq & \left(1+2(1-l)^{2}\right) \delta_{1} \mathbf{E} \int_{D}|\nabla u|^{2} d x-\frac{1}{\rho+1}\left(\int_{0}^{t} g(s) d s\right) \mathbf{E} \int_{D}\left|u_{t}\right|^{\rho+2} d x \\
& +(1-l)\left(2 \delta_{1}+\frac{1}{2 \delta_{1}}\right) \mathbf{E}(g \circ \nabla u)(t)+\frac{g(0)}{4 \delta_{2}}\left(1+\frac{C_{p}}{\rho+1}\right) \mathbf{E}\left(-g^{\prime} \circ \nabla u\right)(t) \\
& +\left[\delta_{2}+c \frac{\delta_{2}}{\rho+1}\left(2\left(\mathcal{E}(0)+E_{1}\right)\right)^{\rho}-\int_{0}^{t} g(s) d s\right] \mathbf{E} \int_{D}\left|\nabla u_{t}\right|^{2} d x
\end{aligned}
$$

Proof Differentiating (3.12) with respect to $t$ and making use of (1.2), we arrive at

$$
\begin{aligned}
\chi^{\prime}(t)= & \int_{D} \nabla u(t) \cdot \int_{0}^{t} g(t-s)(\nabla u(t)-\nabla u(s)) d s d x \\
& -\int_{D}\left(\int_{0}^{t} g(t-s) \nabla u(s) d s\right) \cdot\left(\int_{0}^{t} g(t-s)(\nabla u(t)-\nabla u(s)) d s\right) d x \\
& -\left(\int_{0}^{t} g(s) d s\right) \int_{D}\left|\nabla u_{t}\right|^{2} d x-\int_{D} \nabla u_{t} \cdot \int_{0}^{t} g^{\prime}(t-s)(\nabla u(t)-\nabla u(s)) d s d x \\
& -\frac{1}{\rho+1} \int_{D}\left|u_{t}\right|^{\rho} u_{t} \int_{0}^{t} g^{\prime}(t-s)(u(t)-u(s)) d s d x \\
& -\int_{D} \sigma(x, s) d W_{s} \int_{0}^{t} g(t-s)(u(t)-u(s)) d s d x \\
& -\frac{1}{\rho+1}\left(\int_{0}^{t} g(s) d s\right) \int_{D}\left|u_{t}\right|^{\rho+2} d x .
\end{aligned}
$$

We take the expectation of the above formula to get the following result:

$$
\mathbf{E} \chi^{\prime}(t)=\mathbf{E} \int_{D} \nabla u(t) \cdot \int_{0}^{t} g(t-s)(\nabla u(t)-\nabla u(s)) d s d x
$$




$$
\begin{aligned}
& -\mathbf{E} \int_{D}\left(\int_{0}^{t} g(t-s) \nabla u(s) d s\right) \cdot\left(\int_{0}^{t} g(t-s)(\nabla u(t)-\nabla u(s)) d s\right) d x \\
& -\mathbf{E}\left(\int_{0}^{t} g(s) d s\right) \int_{D}\left|\nabla u_{t}\right|^{2} d x-\mathbf{E} \int_{D} \nabla u_{t} \cdot \int_{0}^{t} g^{\prime}(t-s)(\nabla u(t)-\nabla u(s)) d s d x \\
& -\frac{1}{\rho+1} \mathbf{E} \int_{D}\left|u_{t}\right|^{\rho} u_{t} \int_{0}^{t} g^{\prime}(t-s)(u(t)-u(s)) d s d x \\
& -\frac{1}{\rho+1} \mathbf{E}\left(\int_{0}^{t} g(s) d s\right) \int_{D}\left|u_{t}\right|^{\rho+2} d x .
\end{aligned}
$$

Now we repeat the Cauchy-Schwarz inequality, Hölder's inequality and Young's inequality, to estimate each term on the right-hand side of equation (3.14).

The first item on the right can be estimated as follows:

$$
\begin{aligned}
& \int_{D} \nabla u(t) \cdot \int_{0}^{t} g(t-s)(\nabla u(t)-\nabla u(s)) d s d x \\
& \quad \leq \delta_{1} \int_{D}|\nabla u|^{2} d x+\frac{1-l}{4 \delta_{1}}(g \circ \nabla u)(t), \quad \delta_{1}>0 .
\end{aligned}
$$

As for the second item, we can get the following result from the previously obtained formula (3.10) and $(a+b)^{2} \leq 2\left(a^{2}+b^{2}\right)$ :

$$
\begin{aligned}
& \int_{D}\left(\int_{0}^{t} g(t-s) \nabla u(s) d s\right) \cdot\left(\int_{0}^{t} g(t-s)(\nabla u(t)-\nabla u(s)) d s\right) d x \\
& \leq \delta_{1} \int_{D}\left|\int_{0}^{t} g(t-s) \nabla u(s) d s\right|^{2} d x+\frac{1}{4 \delta_{1}} \int_{D}\left|\int_{0}^{t} g(t-s)(\nabla u(t)-\nabla u(s)) d s\right|^{2} d x \\
& \leq \delta_{1} \int_{D}\left(\int_{0}^{t} g(t-s)(|\nabla u(t)-\nabla u(s)|+|\nabla u(t)|) d s\right)^{2} d x \\
&+\frac{1}{4 \delta_{1}}\left(\int_{0}^{t} g(t-s) d s\right) \int_{D} \int_{0}^{t} g(t-s)|\nabla u(t)-\nabla u(s)|^{2} d s d x \\
& \leq 2 \delta_{1} \int_{D}\left(\int_{0}^{t} g(t-s)|\nabla u(t)-\nabla u(s)| d s\right)^{2} d x \\
& \quad+2 \delta_{1}\left(\int_{0}^{t} g(s) d s\right)^{2} \int_{D}|\nabla u|^{2} d x+\frac{1}{4 \delta_{1}}\left(\int_{0}^{t} g(s) d s\right)(g \circ \nabla u)(t) \\
& \leq \\
& \quad\left(2 \delta_{1}+\frac{1}{4 \delta_{1}}\right)(1-l)(g \circ \nabla u)(t)+2 \delta_{1}(1-l)^{2} \int_{D}|\nabla u|^{2} d x .
\end{aligned}
$$

For the fourth term on the right-hand side of (3.14), it is easy to draw, $\forall \delta_{2}>0$,

$$
\begin{aligned}
& \int_{D} \nabla u_{t}(t) \cdot \int_{0}^{t} g^{\prime}(t-s)(\nabla u(t)-\nabla u(s)) d s d x \\
& \quad \leq \delta_{2} \int_{D}\left|\nabla u_{t}\right|^{2} d x+\frac{g(0)}{4 \delta_{2}} \int_{D} \int_{0}^{t}-g^{\prime}(t-s)|\nabla u(t)-\nabla u(s)|^{2} d s d x
\end{aligned}
$$

For the fifth item, we can similarly get the following results:

$$
\frac{1}{\rho+1} \int_{D}\left|u_{t}\right|^{\rho} u_{t} \int_{0}^{t} g^{\prime}(t-s)(u(t)-u(s)) d s d x
$$




$$
\begin{aligned}
\leq & \frac{1}{\rho+1}\left[\delta_{2} \int_{D}\left|u_{t}\right|^{2(\rho+1)} d x\right. \\
& \left.+\frac{g(0)}{4 \delta_{2}} C_{p} \int_{D} \int_{0}^{t}-g^{\prime}(t-s)|\nabla u(t)-\nabla u(s)|^{2} d s d x\right],
\end{aligned}
$$

where $C_{p}$ is the Poincaré constant and $\delta_{2}>0$. By using the Sobolev embedding

$$
H_{0}^{1}(D) \hookrightarrow L^{2(\rho+1)}(D) \quad \text { for } 0<\rho \leq 2 /(n-2) \text { if } n \geq 3 \text { and } \rho>0 \text { if } n=1,2,
$$

and by (3.7), $\forall t \geq 0$, we get

$$
\int_{D}\left|u_{t}\right|^{2(\rho+1)} d x \leq c\left(2\left(\mathcal{E}(0)+E_{1}\right)\right)^{\rho} \int_{D}\left|\nabla u_{t}\right|^{2} d x
$$

Then (3.18) has the following form:

$$
\begin{aligned}
& \frac{1}{\rho+1} \int_{D}\left|u_{t}\right|^{\rho} u_{t} \int_{0}^{t} g^{\prime}(t-s)(u(t)-u(s)) d s d x \\
& \quad \leq c \delta_{2}\left(2\left(\mathcal{E}(0)+E_{1}\right)\right)^{\rho} \int_{D}\left|\nabla u_{t}\right|^{2} d x+\frac{g(0) C_{p}}{4 \delta_{2}(\rho+1)}\left(-g^{\prime} \circ \nabla u\right)(t) .
\end{aligned}
$$

Combining (3.14)-(3.17) and (3.21), we get (3.13). The proof is completed.

Theorem 3.4 Let $u_{0}(x)$ and $u_{1}(x)$ be $\mathcal{F}_{0}$-measurable with $u_{0}(x) \in L^{2}\left(\Omega ; H_{0}^{1}(D)\right)$ and $u_{1}(x) \in L^{2}\left(\Omega ; L^{2}(D)\right)$. Assume that (A1)-(A3) hold. Then there exist positive constants $k_{1}, k_{2}, k_{3}$, and $\varepsilon_{0}$ such that the solution of (1.2) satisfies

$$
\mathbf{E} \mathcal{E}(t) \leq k_{3} H_{1}^{-1}\left(k_{1} t+k_{2}\right) \quad \forall t \geq 0
$$

where

$$
H_{1}(t)=\int_{t}^{1} \frac{1}{s H_{0}^{\prime}\left(\varepsilon_{0} s\right)} d s \quad \text { and } \quad H_{0}(t)=H(J(t))
$$

provided that $J$ is a positive $C^{1}$ function, with $J(0)=0$, for which $H_{0}$ is a strictly increasing and convex $C^{2}$ function on $(0, r]$ and

$$
\int_{t}^{+\infty} \frac{g(s)}{H_{0}^{-1}\left(-g^{\prime}(s)\right)} d s<+\infty
$$

$H(t)=c t^{p}$, for $1<p<\frac{3}{2}$. Moreover, if $\int_{0}^{t} H_{1}(t) d t<+\infty$ for some choice of $J$, then we have the improved estimate:

$$
\mathbf{E} \mathcal{E}(t) \leq k_{3} G^{-1}\left(k_{1} t+k_{2}\right) \quad \text { where } G(t)=\int_{t}^{1} \frac{1}{s H^{\prime}\left(\varepsilon_{0} s\right)} d s .
$$

\section{Remark 3.1}

1. By using the property of $H$, we can show that the $H_{1}$ function is strictly decremented and raised on $(0,1]$, with $\lim _{t \rightarrow 0} H_{1}(t)=+\infty$. So Theorem 3.4 ensures

$$
\lim _{t \rightarrow 0} \mathcal{E}(t)=0 .
$$


2. Our result is obtained under the very general assumption of the relaxation function $g$, which allows the processing of the larger class function $g$, which guarantees uniform stability of (1.2) and has a decay rate explicit formula energy.

3. The usual exponential and polynomial decay rate estimates have proven that $g$ is satisfied (2.2) and $g^{\prime} \leq-k g^{p}, 1 \leq p<3 / 2$, it is a special case of our results. For these special cases, we will prove that this is a "simple" proof.

4. Our results allow the relaxation function to not necessarily exhibit exponential decay or polynomial decay. For example, if

$$
g(t)=a e^{-t^{q}}
$$

for $0<q<1$ and $a$ is chosen so that $g$ satisfies (2.2), then $g^{\prime}(t)=-H(g(t))$ where, for $t \in(0, r], r<a$,

$$
H(t)=\frac{q t}{[\ln (a / t)]^{\frac{1}{q}-1}},
$$

which satisfies hypothesis (A3). Also, by taking $J(t)=t^{\alpha}$, (3.23) is satisfied with any $\alpha>1$. For this reason, we can use Theorem 3.4 and perform some calculations to infer that the energy is attenuated by the same $g$, i.e.,

$$
\mathbf{E} \mathcal{E}(t) \leq c e^{-k t^{q}}
$$

5. With (A2) and (A3), we can easily infer $\lim _{t \rightarrow \infty} g(t)=0$. This means that $\lim _{t \rightarrow+\infty}\left(-g^{\prime}(t)\right)$ cannot be equal to a positive number, so it is natural to assume $\lim _{t \rightarrow+\infty}\left(-g^{\prime}(t)\right)=0$. Therefore, there is $t_{1}>0$ big enough so that $g\left(t_{1}\right)>0$ and

$$
\max \left\{g(t),-g^{\prime}(t)\right\}<\min \left\{r, H(r), H_{0}(r)\right\}, \quad \forall t \geq t_{1} .
$$

As $g$ is nonincreasing, $g(0)>0$ and $g\left(t_{1}\right)>0$, then $g(t)>0$ for any $t \in\left[0, t_{1}\right]$ and

$$
0<g\left(t_{1}\right) \leq g(t) \leq g(0), \quad \forall t \in\left[0, t_{1}\right] .
$$

Hence, since $H$ is a positive continuous function, then

$$
a \leq H(g(t)) \leq b, \quad \forall t \in\left[0, t_{1}\right]
$$

for some positive constants $a$ and $b$. Consequently, for all $t \in\left[0, t_{1}\right]$,

$$
g^{\prime}(t) \leq-H(g(t)) \leq-a=-\frac{a}{g(0)} g(0) \leq-\frac{a}{g(0)} g(t),
$$

which gives, for some positive constant $d$, we have

$$
g^{\prime}(t) \leq-d g(t), \quad \forall t \in\left[0, t_{1}\right]
$$

Now let us prove Theorem 3.4. 
Proof We consider the functional

$$
L(t)=\mathbf{E}(M \mathcal{E}(t)+\varepsilon \Psi(t)+\chi(t))
$$

where $M$ and $\varepsilon$ are to be specified later. Let $g_{0}=\int_{0}^{t_{1}} g(s) d s$, where $t_{1}>0$ was introduced in (3.25). Using (3.3), (3.8), (3.13), and $\int_{0}^{t} g(s) d s \geq g_{0}, t \geq t_{1}$, we obtain

$$
\begin{aligned}
L^{\prime}(t) \leq & \mathbf{E}\left(\left[\frac{M}{2}-\frac{g(0)}{4 \delta_{2}}\left(1+\frac{C_{p}}{\rho+1}\right)\right]\left(g^{\prime} \circ \nabla u\right)(t)+\frac{\varepsilon-g_{0}}{\rho+1} \int_{D}\left|u_{t}\right|^{\rho+2} d x\right. \\
& -\left[\frac{l}{2} \varepsilon-\left(1+2(1-l)^{2}\right) \delta_{1}\right] \int_{D}|\nabla u|^{2} d x \\
& -\left[g_{0}-\varepsilon-\delta_{2}-c \delta_{2}\left(2\left(\mathcal{E}(0)+E_{1}\right)\right)^{\rho}\right] \int_{D}\left|\nabla u_{t}\right|^{2} d x \\
& +(1-l)\left(\frac{\varepsilon}{2 l}+2 \delta_{1}+\frac{1}{2 \delta_{1}}\right)(g \circ \nabla u)(t) \\
& \left.+\frac{M}{2} \sum_{i=1}^{\infty} \int_{D} \lambda_{i} e_{i}^{2}(x) \sigma^{2}(x, t) d x\right) .
\end{aligned}
$$

At this point, we choose our constant carefully. First, we choose $\varepsilon<g_{0}$, then $\delta_{1}$ and $\delta_{2}$ are small enough so that

$$
\frac{l}{2} \varepsilon-\left(1+2(1-l)^{2}\right) \delta_{1}>0, \quad g_{0}-\varepsilon-\delta_{2}-c \delta_{2}\left(2\left(\mathcal{E}(0)+E_{1}\right)\right)^{\rho}>0 .
$$

Finally, we take $M$ sufficiently large so that

$$
\frac{M}{2}-\frac{g(0)}{4 \delta_{2}}\left(1+\frac{C_{p}}{\rho+1}\right) \geq 0
$$

Therefore, (3.27) reduces to

$$
L^{\prime}(t) \leq-k \mathbf{E} \mathcal{E}(t)+c \mathbf{E}(g \circ \nabla u)(t)+\frac{M}{2} \sum_{i=1}^{\infty} \mathbf{E} \int_{D} \lambda_{i} e_{i}^{2}(x) \sigma^{2}(x, t) d x, \quad \forall t \geq t_{1} .
$$

On the other hand, we can choose $M$ even larger (if needed) so that

$$
L \sim \mathbf{E} \mathcal{E} .
$$

Now,we use (3.3) and (3.26), for any $t \geq t_{1}$,

$$
\begin{aligned}
& \mathbf{E} \int_{0}^{t_{1}} g(s) \int_{D}|\nabla u(t)-\nabla u(t-s)|^{2} d x d s \\
& \quad \leq-\frac{1}{d} \mathbf{E} \int_{0}^{t_{1}} g^{\prime}(s) \int_{D}|\nabla u(t)-\nabla u(t-s)|^{2} d x d s \\
& \quad \leq-c\left(\mathbf{E} \mathcal{E}^{\prime}(t)-\sum_{i=1}^{\infty} \mathbf{E} \int_{D} \lambda_{i} e_{i}^{2}(x) \sigma^{2}(x, t) d x\right) .
\end{aligned}
$$


Next, we take $F(t)=L(t)+c \mathbf{E} \mathcal{E}(t)-(M+2 c) S(t)$, here $M$ was mentioned in (3.27), $S(t)=$ $\frac{1}{2} \sum_{i=1}^{\infty} \mathbf{E} \int_{0}^{t} \int_{D} \lambda_{i} e_{i}^{2}(x) \sigma^{2}(x, s) d x d s$, we assume that $\sigma(x, t)$ satisfies

(A4) $S(t) \leq \frac{1}{M^{\gamma}}, \gamma>1$.

This means that $F(t)$ is equivalent to $\mathbf{E} \mathcal{E}(t)$, therefore, using (3.28)-(3.30), for all $t \geq t_{1}$, we get

$$
F^{\prime}(t) \leq-m \mathbf{E} \mathcal{E}(t)+c \mathbf{E} \int_{t_{1}}^{t} g(s) \int_{D}|\nabla u(t)-\nabla u(t-s)|^{2} d x d s .
$$

In the case of $p=1$, we use estimate (3.31) to get the following result:

$$
\begin{aligned}
F^{\prime}(t) & \leq-m \mathbf{E} \mathcal{E}(t)+c \mathbf{E}(g \circ \nabla u)(t) \\
& \leq-m \mathbf{E} \mathcal{E}(t)-c \mathbf{E} \mathcal{E}^{\prime}(t), \quad \forall t \geq t_{1},
\end{aligned}
$$

which gives

$$
(F+c \mathbf{E} \mathcal{E})^{\prime}(t) \leq-m \mathbf{E} \mathcal{E}(t), \quad \forall t \geq t_{1}
$$

Hence, using the fact that $F+c \mathbf{E} \mathcal{E} \sim \mathbf{E} \mathcal{E}$, we easily obtain

$$
\mathbf{E} \mathcal{E}(t) \leq c^{\prime} e^{-c t}=c G^{-1}(t)
$$

In the case of $1<p<\frac{3}{2}$, one can easily show that $\int_{0}^{+\infty} g^{1-\delta_{0}}(s) d s<+\infty$ for any $\delta_{0}<2-p$. Using (3.3) and (3.7), and choosing $t_{1}$ even larger if needed, we deduce that, for all $t \geq t_{1}$,

$$
\begin{aligned}
\eta(t) & :=\int_{t_{1}}^{t} g^{1-\delta_{0}}(s) \int_{D}|\nabla u(t)-\nabla u(t-s)|^{2} d x d s \\
& \leq 2 \int_{t_{1}}^{t} g^{1-\delta_{0}}(s) \int_{D}\left(|\nabla u(t)|^{2}+|\nabla u(t-s)|^{2}\right) d x d s \\
& \leq c\left(\mathcal{E}(0)+E_{1}\right) \int_{t_{1}}^{t} g^{1-\delta_{0}}(s) d s<1 .
\end{aligned}
$$

Then, Jensen's inequality, (3.3), hypotheses (A2) and (A3), and (3.32) altogether lead to

$$
\begin{aligned}
& \mathbf{E} \int_{t_{1}}^{t} g(s) \int_{D}|\nabla u(t)-\nabla u(t-s)|^{2} d x d s \\
& \quad=\mathbf{E} \int_{t_{1}}^{t} g^{\delta_{0}}(s) g^{1-\delta_{0}}(s) \int_{D}|\nabla u(t)-\nabla u(t-s)|^{2} d x d s \\
& \quad=\mathbf{E} \int_{t_{1}}^{t} g^{\left(p-1+\delta_{0}\right)\left(\frac{\delta_{0}}{p-1+\delta_{0}}\right)}(s) g^{1-\delta_{0}}(s) \int_{D}|\nabla u(t)-\nabla u(t-s)|^{2} d x d s \\
& \quad \leq \mathbf{E}\left(\eta(t)\left[\frac{1}{\eta(t)} \int_{t_{1}}^{t} g(s)^{\left(p-1+\delta_{0}\right)} g^{1-\delta_{0}}(s) \int_{D}|\nabla u(t)-\nabla u(t-s)|^{2} d x d s\right]^{\frac{\delta_{0}}{p-1+\delta_{0}}}\right) \\
& \quad \leq \mathbf{E}\left[\int_{t_{1}}^{t} g(s)^{p} \int_{D}|\nabla u(t)-\nabla u(t-s)|^{2} d x d s\right]^{\frac{\delta_{0}}{p-1+\delta_{0}}}
\end{aligned}
$$




$$
\begin{aligned}
& \leq c \mathbf{E}\left[\int_{t_{1}}^{t}-g^{\prime}(s) \int_{D}|\nabla u(t)-\nabla u(t-s)|^{2} d x d s\right]^{\frac{\delta_{0}}{p-1+\delta_{0}}} \\
& \leq c\left[-\mathbf{E} \mathcal{E}^{\prime}(t)+\sum_{i=1}^{\infty} \mathbf{E} \int_{D} \lambda_{i} e_{i}^{2}(x) \sigma^{2}(x, t) d x\right]^{\frac{\delta_{0}}{p-1+\delta_{0}}} .
\end{aligned}
$$

Then, in particular, for $\delta_{0}=\frac{1}{2}$ we conclude that (3.31) becomes

$$
\begin{aligned}
F^{\prime}(t) \leq & -m \mathbf{E} \mathcal{E}(t)+c\left[-\mathbf{E} \mathcal{E}^{\prime}(t)+\sum_{i=1}^{\infty} \mathbf{E} \int_{D} \lambda_{i} e_{i}^{2}(x) \sigma^{2}(x, t) d x\right]^{\frac{1}{2 p-1}} \\
& +\frac{M}{2} \sum_{i=1}^{\infty} \mathbf{E} \int_{D} \lambda_{i} e_{i}^{2}(x) \sigma^{2}(x, t) d x .
\end{aligned}
$$

Now, we multiply both sides by $(\mathbf{E} \mathcal{E}(t))^{\alpha}$, using $\alpha=2 p-2$ and (3.3) to get

$$
\begin{aligned}
&\left(F(t)(\mathbf{E} \mathcal{E}(t))^{\alpha}\right)^{\prime} \\
&=F^{\prime}(t)(\mathbf{E} \mathcal{E}(t))^{\alpha}+\alpha F(t)(\mathbf{E} \mathcal{E}(t))^{\alpha-1}(\mathbf{E} \mathcal{E}(t))^{\prime} \\
& \leq- m(\mathbf{E} \mathcal{E}(t))^{1+\alpha}+c(\mathbf{E} \mathcal{E}(t))^{\alpha}\left[-\mathbf{E} \mathcal{E}^{\prime}(t)+\sum_{i=1}^{\infty} \mathbf{E} \int_{D} \lambda_{i} e_{i}^{2}(x) \sigma^{2}(x, t) d x\right]^{\frac{1}{1+\alpha}} \\
&+\frac{M}{2} \sum_{i=1}^{\infty} \mathbf{E} \int_{D} \lambda_{i} e_{i}^{2}(x) \sigma^{2}(x, t) d x(\mathbf{E} \mathcal{E}(t))^{\alpha}+\alpha F(t)(\mathbf{E} \mathcal{E}(t))^{\alpha-1}(\mathbf{E} \mathcal{E}(t))^{\prime} .
\end{aligned}
$$

Then, applying Young's inequality, $q=1+\alpha$, and $q^{\prime}=\frac{1+\alpha}{\alpha}$, we get

$$
\begin{aligned}
\left(F(t)(\mathbf{E} \mathcal{E}(t))^{\alpha}\right)^{\prime} \\
\leq-m(\mathbf{E} \mathcal{E}(t))^{1+\alpha}+\varepsilon(\mathbf{E} \mathcal{E}(t))^{1+\alpha}-C_{\varepsilon} \mathbf{E} \mathcal{E}^{\prime}(t)+C_{\varepsilon} \sum_{i=1}^{\infty} \mathbf{E} \int_{D} \lambda_{i} e_{i}^{2}(x) \sigma^{2}(x, t) d x \\
\quad+\alpha F(t)(\mathbf{E} \mathcal{E}(t))^{\alpha-1} \mathbf{E} \mathcal{E}^{\prime}(t)+\frac{M}{2} \sum_{i=1}^{\infty} \mathbf{E} \int_{D} \lambda_{i} e_{i}^{2}(x) \sigma^{2}(x, t) d x(\mathbf{E} \mathcal{E}(t))^{\alpha} \\
\leq-m(\mathbf{E} \mathcal{E}(t))^{1+\alpha}+\varepsilon(\mathbf{E} \mathcal{E}(t))^{1+\alpha}-C_{\varepsilon} \mathbf{E} \mathcal{E}^{\prime}(t)+C_{\varepsilon} \sum_{i=1}^{\infty} \mathbf{E} \int_{D} \lambda_{i} e_{i}^{2}(x) \sigma^{2}(x, t) d x \\
\quad+\frac{\alpha}{1+\alpha}\left((\mathbf{E} \mathcal{E}(t))^{1+\alpha}\right)^{\prime}+\frac{M}{2} \sum_{i=1}^{\infty} \mathbf{E} \int_{D} \lambda_{i} e_{i}^{2}(x) \sigma^{2}(x, t) d x(\mathbf{E} \mathcal{E}(t))^{\alpha} .
\end{aligned}
$$

Let $F_{0}(t)=F(t)(\mathbf{E} \mathcal{E}(t))^{\alpha}-\frac{\alpha}{1+\alpha}(\mathbf{E} \mathcal{E}(t))^{1+\alpha}+C_{\varepsilon} \mathbf{E} \mathcal{E}(t)-2 C_{\varepsilon} S(t)-M\left(\mathbf{E} \mathcal{E}(0)+E_{1}\right)^{\alpha} S(t)$. So choose $\varepsilon<m$ so that

$$
F_{0}^{\prime}(t) \leq-m^{\prime}(\mathbf{E} \mathcal{E}(t))^{1+\alpha}
$$


It can be known from (A4) that it is obviously equivalent to $\mathbf{E} \mathcal{E}(t)$. Therefore we have, for some $a_{0}>0$,

$$
F_{0}^{\prime}(t) \leq-a_{0} F_{0}^{1+\alpha}(t)
$$

from which we easily infer that

$$
\mathbf{E} \mathcal{E}(t) \leq \frac{c}{\left(c^{\prime} t+c^{\prime \prime}\right)^{\frac{1}{2 p-2}}}
$$

By recalling that $p<\frac{3}{2}$ and using (3.33), we find that $\int_{0}^{+\infty} \mathcal{E}(s) d s<+\infty$. Therefore, noting that

$$
\int_{0}^{t} \int_{D}|\nabla u(t)-\nabla u(t-s)|^{2} d x d s \leq c \int_{0}^{t} \mathcal{E}(s) d s
$$

estimate (3.31) gives

$$
\begin{aligned}
F^{\prime}(t) & \leq-m \mathbf{E} \mathcal{E}(t)+c \mathbf{E}\left(g^{p \cdot \frac{1}{p}} \circ \nabla u\right)(t) \leq-m \mathbf{E} \mathcal{E}(t)+c\left[\mathbf{E}\left(g^{p} \circ \nabla u\right)(t)\right]^{\frac{1}{p}} \\
& \leq-m \mathbf{E} \mathcal{E}(t)+c\left[\mathbf{E}\left(-g^{\prime} \circ \nabla u\right)(t)\right]^{\frac{1}{p}} \leq-m \mathbf{E} \mathcal{E}(t)+c\left[-\mathbf{E} \mathcal{E}^{\prime}(t)\right]^{\frac{1}{p}} .
\end{aligned}
$$

Hence, repeating the above steps, with $\alpha=p-1$, we obtain

$$
\mathbf{E} \mathcal{E}(t) \leq \frac{c}{\left(c^{\prime} t+c^{\prime \prime}\right)^{\frac{1}{p-1}}}=c G^{-1}\left(c^{\prime} t+c^{\prime \prime}\right) .
$$

Thus the proof of Theorem 3.4 is completed.

Remark 3.2 In particular, when $p=1$, we also need not assume (A4). That is, taking $F(t)=$ $L(t)+c \mathbf{E} \mathcal{E}(t)$, which is clearly equivalent to $\mathbf{E} \mathcal{E}(t)$, and using (3.28)-(3.30), for all $t \geq t_{1}$, we have

$$
\begin{aligned}
F^{\prime}(t) \leq & -m \mathbf{E} \mathcal{E}(t)+c \mathbf{E} \int_{t_{1}}^{t} g(s) \int_{D}|\nabla u(t)-\nabla u(t-s)|^{2} d x d s \\
& +\left(\frac{M}{2}+c\right) \sum_{i=1}^{\infty} \mathbf{E} \int_{D} \lambda_{i} e_{i}^{2}(x) \sigma^{2}(x, t) d x .
\end{aligned}
$$

Estimate (3.31) yields

$$
\begin{aligned}
F^{\prime}(t) & \leq-m \mathbf{E} \mathcal{E}(t)+c \mathbf{E}(g \circ \nabla u)(t)+\left(\frac{M}{2}+c\right) \sum_{i=1}^{\infty} \mathbf{E} \int_{D} \lambda_{i} e_{i}^{2}(x) \sigma^{2}(x, t) d x \\
& \leq-m \mathbf{E} \mathcal{E}(t)-c \mathbf{E} \mathcal{E}^{\prime}(t)+\left(\frac{M}{2}+c\right) \sum_{i=1}^{\infty} \mathbf{E} \int_{D} \lambda_{i} e_{i}^{2}(x) \sigma^{2}(x, t) d x, \quad \forall t \geq t_{1},
\end{aligned}
$$

which gives

$$
(F+c \mathbf{E} \mathcal{E})^{\prime}(t) \leq-m \mathbf{E} \mathcal{E}(t)+\left(\frac{M}{2}+c\right) \sum_{i=1}^{\infty} \mathbf{E} \int_{D} \lambda_{i} e_{i}^{2}(x) \sigma^{2}(x, t) d x, \quad \forall t \geq t_{1} .
$$


Hence, using the fact that $F+c \mathbf{E} \mathcal{E} \sim \mathbf{E} \mathcal{E}$, it is easy to obtain

$$
\mathbf{E} \mathcal{E}(t) \leq c^{\prime} e^{-c t}+(M+2 c) E_{1}
$$

This means that it is progressively stable and degenerates to $(M+2 c) E_{1}$.

\section{Acknowledgements}

The authors are indebted to the Editor for giving some important suggestions which improved the presentation of this paper.

\section{Funding}

Supported in part by China NSF Grant No. 11501442, the Chinese Scholarship Council (201608615041), the Natural Science Basic Research Plan in Shaanxi Province of China No. 2019JM-283 and No. 2017JM4003, and the Excellent Youth Fund of Xi An University of Science and Technology Grant No. 201YQ2-14, 201YQ3-12.

\section{Availability of data and materials}

No data.

\section{Competing interests}

The authors declare that they have no competing interests.

\section{Authors' contributions}

The article is a joint work of four authors who contributed equally to the final version of the paper. All authors read and approved the final manuscript.

\section{Author details}

'School of Natural and Applied Sciences, Northwestern Polytechnical University, Xi'an, China. ${ }^{2}$ Department of Mathematics, Xi An University of Science and Technology, Xi An, China. ${ }^{3}$ College of Geology and Environment, Xi'an University of Science and Technology, Xi'an, China.

\section{Publisher's Note}

Springer Nature remains neutral with regard to jurisdictional claims in published maps and institutional affiliations.

Received: 25 September 2019 Accepted: 10 March 2020 Published online: 18 March 2020

\section{References}

1. Cavalcanti, M.M., Domingos Cavalcanti, V.N., Ferreira, J.: Existence and uniform decay for nonlinear viscoelastic equation with strong damping. Math. Methods Appl. Sci. 24, 1043-1053 (2001)

2. Zhang, H.M., Yuan, C., Yang, G.S., Wu, L., Peng, C., Ye, W., Shen, Y., Moayedi, H: A novel constitutive modelling approach measured under simulated freeze-thaw cycles for the rock failure. Eng. Comput. (2019). https://doi.org/10.1007/s00366-019-00856-4

3. Pazy, A.: Semigroups of Linear Operators and Applications to Partial Differential Equations. Springer, Berlin (1983)

4. Messaoudi, S.A., Mustafa, M.l.: A general stability result for a quasilinear wave equation with memory. Nonlinear Anal. 14, 1854-1864 (2013)

5. Liu, W: General decay and blow-up of solution for a quasilinear viscoelastic problem with nonlinear source. Nonlinear Anal. 73, 1890-1904 (2010)

6. Song, $\mathrm{H}$.: Global nonexistence of positive initial energy solutions for a viscoelastic wave equation. Nonlinear Anal. $125,260-269(2015)$

7. Cavalcanti, M.M., Domingos Cavalcanti, V.N., Prates Filho, J.S., Soriano, J.A.: Existence and uniform decay rates for viscoelastic problems with nonlinear boundary damping. Differential Integral Equations 14, 85-116 (2001)

8. Chow, P.L.: Asymptotics of solutions to semilinear stochastic wave equations. Ann. Appl. Probab. 16, 757-789 (2006)

9. Chow, P.L.: Asymptotic solutions of a nonlinear stochastic beam equation. Discrete Contin. Dyn. Syst. Ser. B 6, 735-749 (2006)

10. Brzeźniak, Z., Maslowski, B., Seidler, J.: Stochastic nonlinear beam equations. Probab. Theory Related Fields 132, 119-149 (2005)

11. Brzeźniak, Z., Zhu, J.H.: Stochastic nonlinear beam equations driven by compensated Poisson random measures. e-print. arXiv:1011.5377v1

12. Kim, J.U.: On the stochastic wave equation with nonlinear damping. Appl. Math. Optim. 58, 29-67 (2008)

13. Barbu, V., Prato, G.D., Tubaro, L.: Stochastic wave equations with dissipative damping. Stochastic Process. Appl. 117, $1001-1013$ (2007)

14. Gao, H.J., Liang, F., Guo, B.L.: Stochastic wave equations with nonlinear damping and source terms. Infin. Dimens. Anal. Quantum Probab. Relat. Top. 16, Article ID 1350013 (2013)

15. Taniguchi, T.: Explosion of solutions to nonlinear stochastic wave equations with multiplicative noise. Nonlinear Anal. 117, 47-64 (2015)

16. Carmona, R., Nualart, D.: Random non-linear wave equation: smoothness of solutions. Probab. Theory Related Fields 95, 87-102 (1993)

17. Crauel, H., Debussche, A., Flandoli, F.: Random attractors. J. Dynam. Differential Equations 9, 307-341 (1997) 
18. Dalang, R., Frangos, N.: The stochastic wave equation in two spatial dimensions. Ann. Probab. 26(I), 187-212 (1998)

19. Millet, A., Morien, P.L.: On a nonlinear stochastic wave equation in the plane: existence and uniqueness of the solution. Ann. Appl. Probab. 11, 922-951 (2001)

20. Parshad, R.D., Beauregard, M.A., Kasimov, A., Said-Houari, B.: Global existence and finite blow-up in a class of stochastic nonlinear wave equations. Commun. Stoch. Anal. Anal.8, 381-411 (2014)

21. Wei, T.T., Jiang, Y.M.: Stochastic wave equations with memory. Chin. Ann. Math. 31B, 329-342 (2010)

22. Liang, F., Gao, H.J.: Explosive solutions of stochastic viscoelastic wave equations with damping. Rev. Math. Phys. 23, 883-902 (2011)

23. Liang, F., Gao, H.J.: Stochastic nonlinear wave equation with memory driven by compensated Poisson random measures. J. Math. Phys. 55, Article ID 033503 (2014)

24. Liang, F., Guo, Z.H.: Asymptotic behavior for second order stochastic evolution equations with memory. J. Math. Anal. Appl. 419, 1333-1350 (2014)

25. Kim, S., Park, J.Y., Kang, Y.H.: Stochastic quasilinear viscoelastic wave equation with nonlinear damping and source terms. Bound. Value Probl. 2018, 14 (2018)

26. Chow, P.L.: Stochastic Partial Differential Equations. Chapman \& Hall/CRC, London (2007)

27. Da Prato, G., Zabczyk, J.: Stochastic Equations in Infinite Dimensions. Cambridge University Press, Cambridge (1992)

\section{Submit your manuscript to a SpringerOpen ${ }^{\circ}$} journal and benefit from:

- Convenient online submission

Rigorous peer review

Open access: articles freely available online

High visibility within the field

- Retaining the copyright to your article

Submit your next manuscript at $\gg$ springeropen.com 